\title{
On group velocity delays in microwave millisecond oscillating events and radio spikes
}

\author{
L. V. Yasnov ${ }^{1}$ and M. Karlický ${ }^{2}$ \\ 1 Radiophysical Research Institute of St.-Petersburg State University, St.-Petersburg, Russia \\ 2 Astronomical Institute of the Academy of Sciences of the Czech Republic, 25165 Ondřejov, Czech Republic
}

Received 17 February 2003 / Accepted 12 June 2003

\begin{abstract}
Time delays between opposite polarizations, interpreted by a difference between the ordinary and extraordinary modes, are studied. Two different density and magnetic field models are used, and three emission mechanisms generating radio waves in double upper-hybrid, plasma, and gyro frequencies are considered. It is found that time delays and their spectra can reach various values and forms depending on plasma parameters in the radio wave emission and propagation. For the emission in double upper-hybrid frequency the considerable decrease of the time delay is due to an increase of the electron plasma density in the radio wave generation. In a dense flare plasma the power index $a$ of the time delay spectrum is negative. On the other hand, in a diluted plasma $a$ becomes positive, but in this case it is much lower than observed for dm-pulsations $(a=3)$. Furthermore model values are compared with the time delays presented by Fleishman et al. (2002), and it is shown that the best agreement is with the model assuming the emission on double gyro-frequency. But, in this case the gyro-resonant absorption limits the angle for escape of radio waves to $\Theta<3-10^{\circ}$. Finally, an effect of the difference of the group and light velocities on the frequency drift is analyzed. It is shown that such an effect can be important for wave propagation along magnetic field lines at frequencies close to the plasma frequency.
\end{abstract}

Key words. plasmas - Sun: flares - Sun: radio radiation

\section{Introduction}

Decimetric and microwave solar radio bursts frequently produce millisecond fine structures (Slottje 1981; Gudel \& Benz 1988; Allaart et al. 1990; Isliker \& Benz 1994; Jiřička et al. 2001; Fu et al. 2000). Some of them appear as quasiperiodic pulsations. The short time scale of the pulsations can be naturally interpreted in terms of the pulsating mode of the underlying coherent emission mechanism (Aschwanden 1987).

This paper analyzes events with a time delay between opposite polarizations. The group delay effect has been studied for solar radio spikes. Benz \& Pianezzi (1997) found a rather small delay (of the order of $100 \mu \mathrm{s}$ ) between different spike polarizations. They concluded that radio spikes are generated in their source as fully polarized while the observed polarization degree arises in a different region in the corona where the plasma frequency and gyrofrequency are much less than the spike frequency. Zlobec \& Karlický (1998) and Wang et al. (2000) reported delays for solar radio spikes up to 2-8 ms. Zlobec \& Karlický (1998) assumed that the time delay can be explained by different trajectories with different lengths for ordinary (o) and extraordinary (x) modes. Chernov \& Zlobec (1995) reported larger delays for fiber bursts and zebras up to $0.1 \mathrm{~s}$. They supposed that the mode coupling and the reflection

Send offprint requests to: L. V. Yasnov, e-mail: karlicky@asu.cas.cz of the original radio signal can generate the delay. But it is not clear how the mode coupling can cause the time delay. For processes connected with the wave reflection in deeper atmospheric layers, in principle, they can produce these time delays, but in this case reflected radio waves are strongly attenuated at layers close to the reflection.

Fleishman et al. (2002) analyzed two events demonstrating periodic narrowband millisecond pulsations of radio emission both in intensity and polarization degree. Large time delays between L- and R-polarization components were found, of $20 \mathrm{~ms}$. The radio emission was shown to be generated as unpolarized by a plasma mechanism at the second harmonic of the upperhybrid frequency. Observed oscillations of the radiation polarization degree arose due to a delay between group velocities of the extraordinary and ordinary modes along the propagation path. The simple theoretical model predicted a dependence of the group delay on frequency as $f^{-3}$.

A delay between ordinary and extraordinary waves must arise in magnetized plasma due to the wave dispersion providing different values of the group velocities of $\mathrm{o}-$ and $\mathrm{x}$-waves.

The expected group delay between the two magnetoionic modes at the frequency $\omega$ is easy to find from the general expression

$\tau=\int_{s_{0}}^{\infty}\left(\frac{1}{v_{\mathrm{x}}}-\frac{1}{v_{\mathrm{o}}}\right) \mathrm{d} s$ 
where $v_{\mathrm{x}}, v_{\mathrm{o}}$ are the group velocities of the extraordinary and ordinary waves, respectively; $s$ is the coordinate along the propagation path, $s_{0}$ is the coordinate of the wave generation region. A full integration of the integral (1) in specific models of the solar atmosphere has not been made up to now. In previous studies, for simplicity, this integral has been only estimated by a multiplication of the term $\left(1 / v_{\mathrm{x}}-1 / v_{\mathrm{o}}\right)$ by an effective scale of the gradual coronal inhomogeneity, neglecting the frequency dependence. Computations of the group delay using this method have been made, e.g. in the paper by Fleishman et al. (2002). They assumed that the radio emission is generated on a fixed frequency in a homogenous source and that is why this model is not suitable for the analysis of spectral characteristics of the time delays. In the following paper the authors (Melnikov et al. 2002) tried to include the effect of a source inhomogeneity on spectral characteristics of the time delay, but without specific models of the solar atmosphere.

In the present paper we compute the time delay of ordinary and extraordinary polarized components of the radio emission caused by a difference of their group velocities in various models of the solar atmosphere. For the first time the dependence of $s_{0}$ (see Eq. (1)) on radiation mechanism and frequency are considered.

\section{Basic equations}

The group velocities of electromagnetic waves can be expressed as (Zheleznyakov 1996):

$v_{\mathrm{o}, \mathrm{x}}=\frac{c}{\frac{\partial \omega \operatorname{Re}\left(n_{0, \mathrm{x}}\right)}{\partial \omega}}$,

where $c$ is the speed of light and

$n_{\mathrm{o}, \mathrm{x}}^{2}=1-\frac{v B}{(-1)^{w}\left(\frac{u^{2}}{4} \sin ^{4} \theta+u B^{2} \cos ^{2} \theta\right)^{0.5}+B-\frac{u}{2} \sin ^{2} \theta}$,

$v=\omega_{\mathrm{p}}^{2} / \omega^{2}, u=\omega_{B}^{2} / \omega^{2}, B=1+i z-v, z=v / \omega, v$ is the electron collision frequency, $\omega_{\mathrm{p}}, \omega_{B}$ are the electron plasma and gyro frequencies, $\theta$ is the angle between the propagation path and magnetic field, $w=1$ for $\mathrm{x}$-mode and $w=2$ for o-mode. If $v \ll \omega$ then

$v_{\mathrm{o}, \mathrm{x}}=\frac{c d\left(2-2 v^{2}+(-1)^{w} d-u \sin ^{2} \theta\right)^{3 / 2} \sqrt{R}}{A}$,

where

$$
\begin{aligned}
& R=2-6 v^{2}+4 v^{4}+(-1)^{w} d-u \sin ^{2} \theta, \\
& A=-2(-1)^{w} u\left(-2\left(1-v^{2}\right)^{2} \cos ^{2} \theta\left(4-5 v^{2}+v^{4}-2 u \sin ^{2} \theta\right)\right. \\
& \left.+u \sin ^{4} \theta\left(-2+3 v^{2}+u \sin ^{2} \theta\right)\right)+d\left(4\left(1-v^{2}\right)^{2}\right. \\
& \left.+4 u\left(1-v^{2}\right)^{2} \cos ^{2} \theta+u \sin ^{2} \theta\left(-4+6 v^{2}+2 u \sin ^{2} \theta\right)\right), \\
& d=\sqrt{u\left(4\left(1-v^{2}\right)^{2} \cos ^{2} \theta+u \sin ^{4} \theta\right)} .
\end{aligned}
$$

In Fig. 1 the dependencies of the ratio of group and light velocities on frequency, computed using the relation (2), are shown.
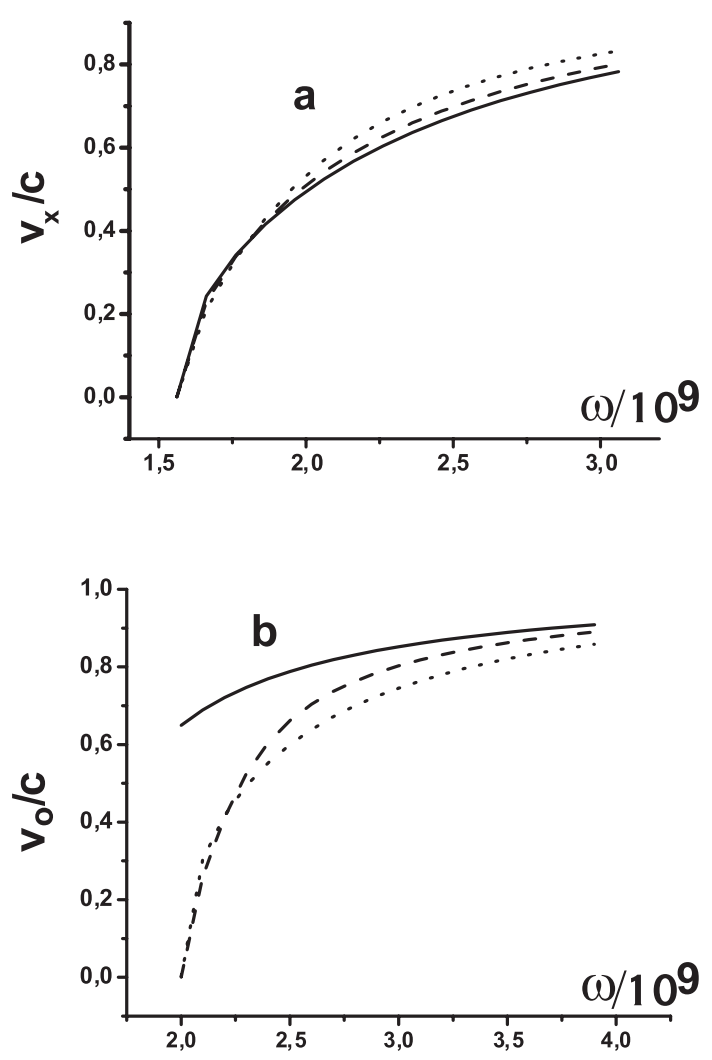

Fig. 1. The dependence of the ratio of group and light velocities on frequency: a) for the extraordinary mode, b) for the ordinary mode. The solid line $-\theta=0$, the dashed line $-\theta=45^{\circ}$, and dotted line $\theta=90^{\circ}$. In the computations $\omega_{\mathrm{p}}=2 \times 10^{9} \mathrm{~s}^{-1}, \omega_{B}=10^{9} \mathrm{~s}^{-1}$ were used.

Real values of velocities for the extraordinary mode are obtained for frequencies $\omega \geq \frac{1}{2}\left(\sqrt{\omega_{\mathrm{p}}^{2}+4 \omega_{B}^{2}}+\omega_{B}\right)$, and for the ordinary mode for $\omega \geq \omega_{\mathrm{p}}$; in both cases for several values of the angle $\theta$.

It can be seen that the group velocity of the ordinary mode close to the limit frequency increases considerably in a transition to the quasi-parallel wave propagation. In the following computations the dependence of the group velocity on the angle $\theta$ is considered.

In the quasi-parallel case, i.e. when the following conditions are fulfilled

$\frac{u \sin ^{4} \theta}{4 \cos ^{2} \theta} \ll(1-v)^{2}$

$\left|1+(-1)^{w}\right| \sqrt{u} \cos \theta \| \gg \frac{u \sin ^{2} \theta}{|1-v|}$,

the group velocities can be expressed as

$v_{\mathrm{o}, \mathrm{x}}=\frac{2 c\left(1+(-1)^{w} \sqrt{u} \cos \theta\right)^{2} \sqrt{1-\frac{v^{2}}{1+(-1)^{w} \sqrt{u} \cos \theta}}}{2-(-1)^{w} \sqrt{u}\left(-4+v^{2}\right) \cos \theta+2 u \cos ^{2} \theta}$.

The approximate values of the group velocities valid for $\omega^{2} \geq$ $\omega_{\mathrm{p}}^{2}, \omega_{B}^{2}$ can be derived as follows:

$v_{\mathrm{x}, \mathrm{o}} \approx \frac{c}{1+v-(-1)^{w} v \sqrt{u} \cos \theta}$. 
Equation (1) together with Eq. (3) provides

$\tau=\frac{2}{c} \int_{s_{0}}^{\infty} v \sqrt{u} \cos \theta \mathrm{d} s$.

If the dependence of $s_{0}$ on frequency is neglected then from the relation (4) it follows that the time delay is proportional to $\omega^{-3}$. But $s_{0}$ is a function of the frequency. The position $s_{0}$ can be derived solving one of the following equations:

$n \omega_{\mathrm{p}}\left(s_{0}\right)=\omega$,

$n \omega_{B}\left(s_{0}\right)=\omega$,

$n \sqrt{\omega_{\mathrm{p}}^{2}\left(s_{0}\right)+\omega_{B}^{2}\left(s_{0}\right)}=\omega$,

for various types of plasma emission and the equation

$\tau_{\mathrm{x}, \mathrm{o}}\left(\omega, s_{0}\right) \approx 1$

for the gyro-synchrotron emission, where $\tau_{\mathrm{x}, \mathrm{o}}$ is the optical depth and $n$ is the harmonic number.

Now, let us consider some specific parametric models of the solar atmosphere and some specific mechanisms of the radio wave generation. Based on these models and general formulas (1 and 2) we will determine the solar plasma parameters in space of electromagnetic wave propagation and the time delay for various wavelengths, i.e. the delay spectrum will be constructed.

In the first model the magnetic field induction $B$ is taken in the form of the vertical dipole, which is situated under the photosphere at the depth $r_{0}$ (see e.g. Gurman \& House 1981)

$B(h, y)=B_{0}(2 r)^{3}\left(\left(h+r_{0}\right)^{2}+y^{2}\right)^{-3} \sqrt{4\left(h+r_{0}\right)^{2}+y^{2}}$.

Then its component along $h$ is

$B_{h}(h, y)=B_{0}(2 r)^{3}\left(\left(h+r_{0}\right)^{2}+y^{2}\right)^{-5 / 2}\left(2\left(h+r_{0}\right)^{2}-y^{2}\right)$,

where $h$ is the height above the photosphere, $y$ is the coordinate along the solar surface, $B_{0}$ is the induction at distance $y_{\max }=2 r$. In computations the parameters were taken as: $r=5 \times 10^{9} \mathrm{~cm}, r_{0}=5 \times 10^{9} \mathrm{~cm}, y=0.1 y_{\max }, B_{0}=10 \mathrm{G}$, which corresponds to the magnetic field induction of $2500 \mathrm{G}$ at the photospheric level. In other models the magnetic field was computed according to the formula by Dulk \& McLean (1978)

$B(h)=0.5\left(\frac{h(\mathrm{~cm})}{7 \times 10^{10}}\right)^{-1.5}(\mathrm{G})$,

which is valid for heights $h=1.5 \times 10^{9}-7 \times 10^{11} \mathrm{~cm}$. On the other hand, the electron density $n$ was described using the formula by Newkirk (1967), which includes a multiplication parameter $k$

$n(h)=k .8 .4 \times 10^{4} \times 10^{\left(4.32 /\left(1+h / r_{\mathrm{s}}\right)\right)}$,

where $r_{\mathrm{s}}=7 \times 10^{10} \mathrm{~cm}$. Furthermore, the barometric law of the electron density (or the plasma frequency) on height was considered as

$f_{\mathrm{p}}=f_{\mathrm{p} 0} \exp \left(-\frac{h-h_{0}}{9 \times 10^{3} T_{\mathrm{e}}}\right) \sqrt{k}$, where $h_{0}$ is the height at which the plasma frequency equals to $2 \pi f_{\mathrm{p} 0}$. In agreement with Bogod et al. (2001) we take $f_{\mathrm{p} 0}=$ $10^{9} \mathrm{~Hz}$ at $h_{0}=1.5 \times 10^{9} \mathrm{~cm}$, and $T_{\mathrm{e}}=2 \times 10^{7} \mathrm{~K}$. Putting the relations (7)-(10) into the Eqs. (5), the position $s_{0}$ is determined and then the integral (1) can be computed. The integration is made along the height $h(s=h)$. In the first magnetic field model (relation (7)) the angle between the wave propagation direction and the magnetic field along the integration path is determined according to the relation

$\theta(h)=\arccos \left(\frac{2\left(h+r_{0}\right)^{2}-y^{2}}{\sqrt{\left(h+r_{0}\right)^{2}+y^{2}} \sqrt{4\left(h+r_{0}\right)^{2}-y^{2}}}\right)$.

On the other hand, in the model expressed by the relation (8) the angle $\theta=0$ is assumed. Computations are performed in the range of frequencies $2.81-2.89 \mathrm{GHz}$, because in this range Fleishman et al. (2002) found results important for our study.

\section{Radio emission on double upper-hybrid frequency}

Some theories of nonthermal emission are based on the electrostatic wave emission from a source at the double plasma resonance, i.e. the upper hybrid frequency $\omega_{\mathrm{UH}}$ equals some harmonic of the electron cyclotron frequency: $\omega_{\mathrm{UH}}=\sqrt{\omega_{\mathrm{P}}^{2}\left(s_{0}\right)+\omega_{B}^{2}\left(s_{0}\right)}=n \omega_{B}$ (Zheleznykov \& Zlotnik 1975; Kuijpers 1975; Mollwo 1983; Winglee \& Dulk 1986). Simultaneously the case of a low-anisotropic plasma $\left(\omega_{\mathrm{p}} \geq \omega_{B}\right)$ is assumed. In these conditions the kinetic instability has the lowest threshold for a concentration of hot particles unstable at velocities which are perpendicular to the magnetic field. If conditions of the double plasma resonance are fulfilled then narrowband emissions are generated, which are observed e.g. in the case of spikes and pulsations. Therefore this type of emission will be studied in detail.

In low-anisotropic plasma an escape of the extraordinary mode in the first harmonic of plasma frequencies $\omega_{\mathrm{p}}$ and $\omega_{B}$ is not possible (the refraction index is imaginary). At the first harmonic of the upper-hybrid frequency $\omega_{\mathrm{UH}}$ the escape is possible, but only in the narrow range of angles $\theta$ close to $\pi / 2$. Therefore, in the following the generation of radio waves in the second harmonic of plasma frequencies will be considered $(n=2)$.

Computed results of the time delays $\tau$ and corresponding power indexes $a$ for various atmospheric densities and magnetic fields are plotted in Fig. 2. While the time delay is computed for $2.81 \mathrm{GHz}$, the power index was determined in the 2.81-2.89 GHz range. Values of $k$ are hereinafter limited to $\omega_{\mathrm{p}}>\omega_{B}$ at heights $s_{0}$.

It can be seen in Fig. 2, for the model 1 (solid line), that in dense flare plasmas, when $k>2-3$, the power index $a$ is negative. On the other hand, in dilute plasmas the index $a$ becomes positive, but in this case is considerably lower than that predicted from the simple formula $\tau=\left(1 / v_{\mathrm{x}}-1 / v_{\mathrm{o}}\right) \times$ const $(a=3)$ and lower than those observed in dm-pulsations $(a=3)$ (Fleishman et al. 2002). In the model 2 (dashed line) the time delay $\tau=5 \mathrm{~ms}$ (for observed time delays see Introduction) corresponds to $k=4.5$. The time delay $\tau=20 \mathrm{~ms}$ is not at 

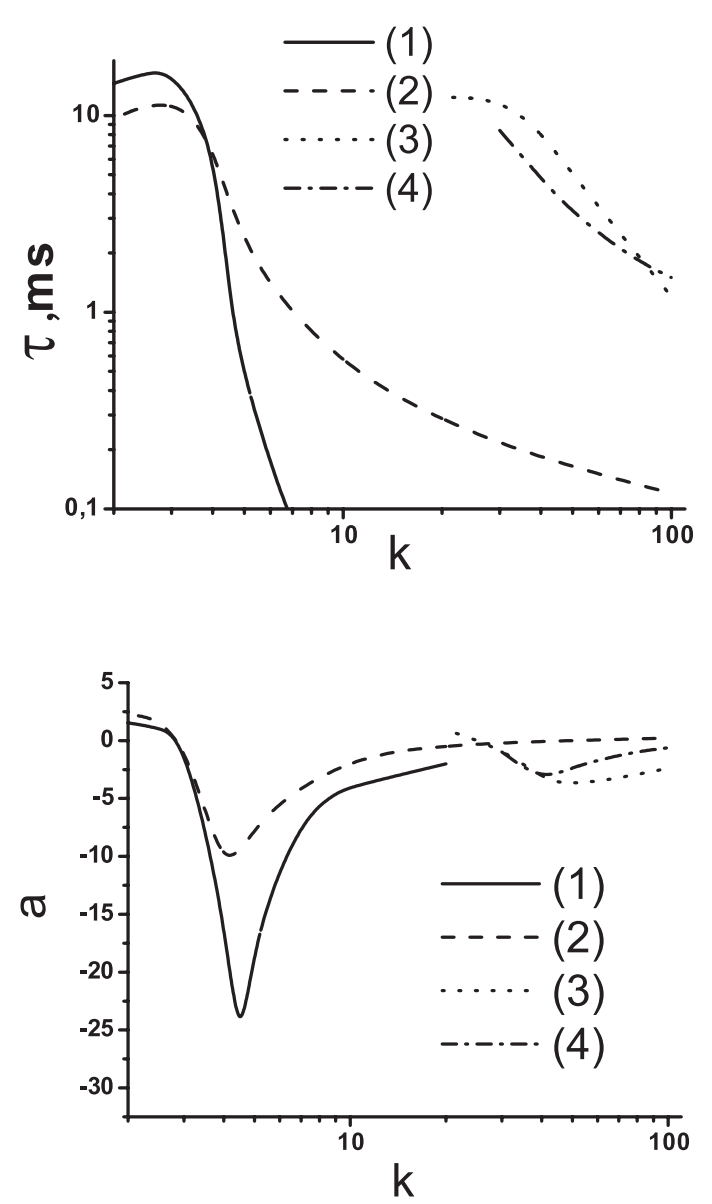

Fig. 2. The time delay $\tau$ and the power index $a$ in the function $\tau \approx$ $1 / \omega^{a}$ vs. the electron density expressed through the multiplication parameter $k$ : (1) in the barometric solar atmosphere (relation (10)) with the dipole magnetic field (relation (7)); (2) in the barometric solar atmosphere (10) with the magnetic field described by formula (8) (Dulk \& McLean 1978); (3) in the atmosphere where the electron density is described according to the Newkirk formula (9) and the magnetic field by the dipole one (relation (7)); and (4) in the atmosphere with the electron density according to the Newkirk formula (9) and the magnetic field expressed according to (8). The observed values of $\tau=20 \mathrm{~ms}, 5 \mathrm{~ms}, 100 \mu \mathrm{s}$ (see Introduction).

all reached in this model. On the other hand, in the model 3 (dotted line) the time delay $\tau=5 \mathrm{~ms}$ corresponds to $k=45$. Similarly, the time delay $\tau=20 \mathrm{~ms}$ cannot be reached in the model 3. Finally, in the model 4 the time delays $\tau=100 \mu \mathrm{s}$ and $\tau=20 \mathrm{~ms}$ cannot be reached. The value $\tau=5 \mathrm{~ms}$ can be found for $k=40$. Computed values are limited to $k>30$ as only in this case is the radio emission generated at heights $h>1.5 \times 10^{9} \mathrm{~cm}$, for which the formula (8) is valid.

\section{Radio emission on double plasma frequency}

One of the most popular plasma mechanism used is that in which two oppositely directed Langmuir plasmons produce the radio emission on double plasma frequency (see e.g. Ginzburg \& Zheleznaykov 1958; Melrose 1970). The results of computations of the time delay $\tau$ and the power index $a$ for this emission mechanism in the barometric solar atmosphere with the dipole magnetic field are shown in Fig. 3.
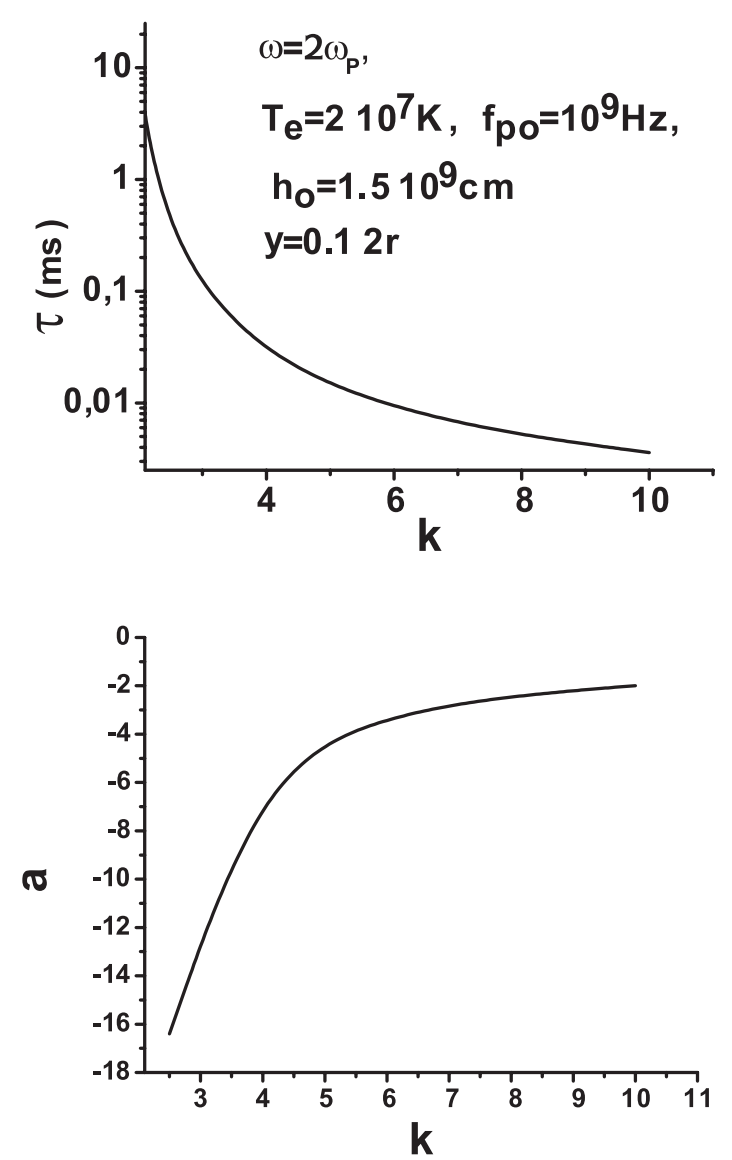

Fig. 3. The time delay $\tau$ and the power index $a$ vs. the electron density in the barometric solar atmosphere expressed by the multiplication parameter $k$, with the dipole magnetic field (the relation (7)).

For this emission mechanism the time delays $\tau=100 \mu \mathrm{s}$ and $5 \mathrm{~ms}$ correspond to $k=3.2$ and 2.0, respectively. Thus, observed time delays can be reached by a variation of the electron density in the solar atmosphere. As in previous cases a decrease of the time delay needs to increase the electron density. It is important to mention that in computations, neglecting the generation position change depending on frequency $\left(s_{0} \equiv s_{0}(\omega)\right)$, the results would be opposite. Furthermore, the time delay $\tau=20 \mathrm{~ms}$ cannot be reached in this model. The power index is here always negative, which is in contradiction to the results shown by Fleishman et al. (2002).

\section{Radio emission on double gyro-frequency}

This emission is connected with a generation of longitudinal electron cyclotron waves or so-called Bernstein modes, which propagate in the direction perpendicular to the magnetic field. As in the previous case, these waves are effectively generated under the condition of $\omega_{\mathrm{p}}>\omega_{B}$ (Bernstein 1958). The corresponding radio waves are then generated due to the coalescence of Bernstein modes. In this case the frequency of the radio emission can be $\omega=2 \omega_{B}$. These processes were used for an explanation of some radio fine structures of type IV radio bursts (see Zheleznyakov \& Zlotnik 1975). If the wavelength of these waves is much greater than the gyro-radius of thermal electrons then their frequency is close to the harmonics of the 
gyrofrequency. Computations of the emission on the frequency $2 \omega_{B}$ in the barometric solar atmosphere with the multiplication parameter $k$ and the dipole magnetic field at $y=0.1 \times 2 r$ show that the time delay depends linearly on $k$ :

$\tau(\mathrm{ms})=7.3 k$,

and the power index is constant and equals 2.26. This simple linear dependence follows from the relation (4). Results are weakly dependent on $y$, e.g. the power index at $y=0$ equals 2.31. The time delay increases not more than $3 \%$. Values of $k$ are limited $(>1.9)$ to performance of the condition $\omega_{\mathrm{p}}>\omega_{B}$. For $k \simeq 3$ the time delay is close to that $(20 \mathrm{~ms})$ presented by Fleishman et al. (2002). Note that the value of $k$ is lower than that given by the limit $2 \omega_{B}>\omega_{\mathrm{p}}$ which corresponds to $k<7.7$. Similar values are obtained also in the model with the barometric atmosphere and the magnetic field described according to the formula by Dulk \& McLean (1978). In this case, the time delay is

$\tau(\mathrm{ms})=4.8 k$,

and the power index is also constant and equals 2.63 (here $1.8<$ $k<7.4)$. In this model the time delay of $20 \mathrm{~ms}$ is obtained for $k=4.2$. Smaller observed values of the time delay $(5 \mathrm{~ms}$ and $100 \mu \mathrm{s}$ ) cannot be reached in this model. If the electron density is given by the Newkirk model (Newkirk 1967) then time delays decrease nearly one order of magnitude: $\tau(\mathrm{ms})=$ $0.69 k, a=2.05$ for the dipole magnetic field and $\tau(\mathrm{ms})=$ $0.46 k, a=2.45$ for the magnetic field according to Dulk \& McLean (1978).

\subsection{Diagnostic statements}

Considering that power indexes for the mentioned time delays are close to those observed $(3 \pm 1)$ it is possible to conclude that the analyzed emission mechanism agrees with the observational results presented by Fleishman et al. (2002). In such a situation, some characteristics of the pulsation source, which was analyzed in the paper by Fleishman et al. (2002), can be derived.

The electron density model with the barometric law is preferred because in the model by Newkirk (1967) the relatively high values of $k=30-40$ are needed for $\tau=20 \mathrm{~ms}$. Taking into account the low frequency part of the event, i.e. $\omega=2 \pi \times 2.81 \times$ $10^{9} \mathrm{~s}^{-1}$, we can estimate the magnetic field (from $\omega=2 \omega_{B}$ ) as $B=m c \omega /(2 e)=500 \mathrm{G}$. The magnetic field changes $e$-times within the distance scale of $L_{B}=1.6 \times 10^{9} \mathrm{~cm}$. If the relation (11) is used then $k=2.7$. In the chosen model (10) it means that the emission is generated at height $h=1.1 \times 10^{9} \mathrm{~cm}$, where the density is $n=3.35 \times 10^{10} \mathrm{~cm}^{-3}$.

Because the averaged polarization degree is very small we must conclude that the wave absorption in layers above the source is negligible. At the layer with $B=500 \times 2 / 3 \mathrm{G}$, a strong absorption on the third harmonic of the gyrofrequency can be expected. Here, at the height of $h=1.6 \times 10^{9} \mathrm{~cm}$ derived from (7), the electron density is $n=3.33 \times 10^{10} \mathrm{~cm}^{-3}$. Using these plasma parameters and the formulas presented in the paper by Zheleznyakov (1977), the optical thickness of the

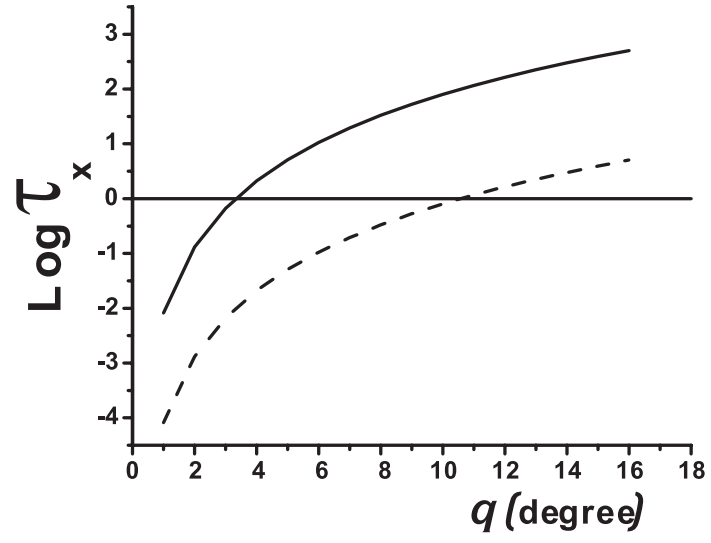

Fig. 4. The optical thickness of the extraordinary mode $\tau_{\mathrm{x}}$ vs. angles $\Theta$ between the line of sight and the magnetic field. The dashed line is for $T_{\mathrm{e}}=2 \times 10^{6} \mathrm{~K}$ and solid line for $T_{\mathrm{e}}=2 \times 10^{7} \mathrm{~K}$.

extraordinary mode due to gyroresonant absorption $\tau_{\mathrm{x}}$ depending on angles between the line of sight and magnetic field $\Theta$ was calculated and the results are shown in Fig. 4. It can be seen that the emission polarization for $T_{\mathrm{e}}=2 \times 10^{7} \mathrm{~K}$ will be low only for angles $\Theta<3^{\circ}$. This puts a strong limit for the validity of this model. A little bit better situation exists for $T_{\mathrm{e}}=2 \times 10^{6} \mathrm{~K}$. In this case, the coefficient of linearity in the relation (11) changes slightly to 6.2 , and the range of the radiation escape increases to angles $\Theta=10^{\circ}$. In the specific interval of angles the optical thickness of the ordinary mode is of 45 orders of magnitude lower than the presented extraordinary one.

\section{Frequency drift}

The frequency drift estimated from spectrograms (for example, using two values of frequency $\omega_{1,2}$ and time $t_{1,2}$ for some radio bursts) depends on the source parameters as follows

$\frac{\omega_{2}-\omega_{1}}{t_{2}-t_{1}}=\frac{\omega_{2}-\omega_{1}}{\int_{s_{0}\left(\omega_{2}\right)}^{s_{\mathrm{r}}} \frac{1}{v_{\mathrm{o}, \mathrm{x}}} \mathrm{d} s-\int_{s_{0}\left(\omega_{1}\right)}^{s_{\mathrm{r}}} \frac{1}{v_{\mathrm{o}, \mathrm{X}}} \mathrm{d} s+\frac{s_{0}\left(\omega_{2}\right)-s_{0}\left(\omega_{1}\right)}{v_{\mathrm{s}}}}$

where $s_{\mathrm{r}}$ is the receiver coordinate, $v_{\mathrm{s}}$ is the velocity of the moving source. The strongest effect on the frequency drift of a difference of the group velocity from the velocity of light will be in the case of the emission on the fundamental plasma frequency $\omega_{\mathrm{p}}$. Therefore, let us consider this case. An observer records the ordinary mode only, even in the case when radio waves propagate along the electron density gradient. Namely, the index of refraction for the extraordinary mode is imaginary in this case. Now, using the model of active regions described by Eqs. (7) and (10) for $y=0$, let us compute the difference of integrals in the denominator of the relation (12), designated as $t_{\mathrm{gr}, 2}-t_{\mathrm{gr}, 1}$, vs. $\theta$. For simplicity, we assume that the magnetic field intensity depends on the height $h$ only, and the angle $\theta$ between the vertical direction and magnetic field lines is constant (i.e. not dependent on $h$ ). As in the previous case we use $\omega_{1}=2 \pi \times 2.81 \times 10^{9} \mathrm{~s}^{-1}, \omega_{2}=2 \pi \times 2.86 \times 10^{9} \mathrm{~s}^{-1}$, and $k=1$. The results are shown in Fig. 5 .

Here, it can be seen that for the propagation of electromagnetic waves along the magnetic field some frequencytime structures with a positive frequency drift can appear 


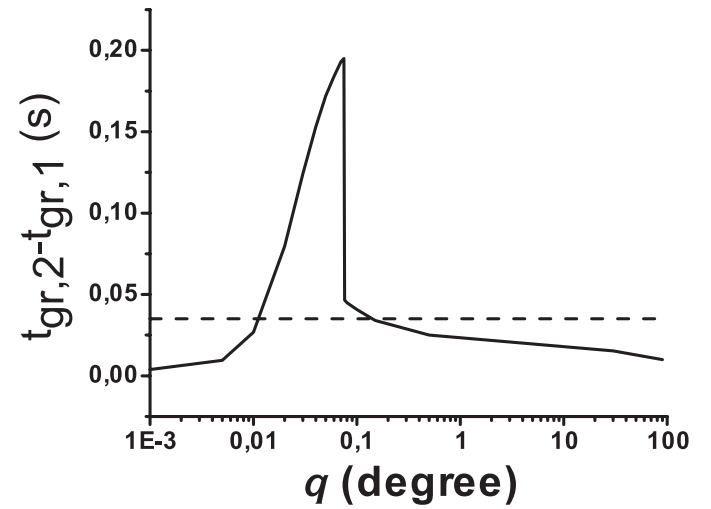

Fig. 5. The difference of integrals in the relation (12) $t_{\mathrm{gr}, 2}-t_{\mathrm{gr}, 1} \mathrm{vs}$. the angle $\theta$ between the propagation direction and the magnetic field. The model of the active region is described by the Eqs. (7) and (10) for the position $y=0$. The horizontal line corresponds to $\mid\left(s_{0}\left(\omega_{2}\right)-\right.$ $\left.s_{0}\left(\omega_{1}\right)\right) / v_{\mathrm{s}} \mid$ for $v_{\mathrm{s}}=0.3 \mathrm{c}$, along $h$ in the observer direction.

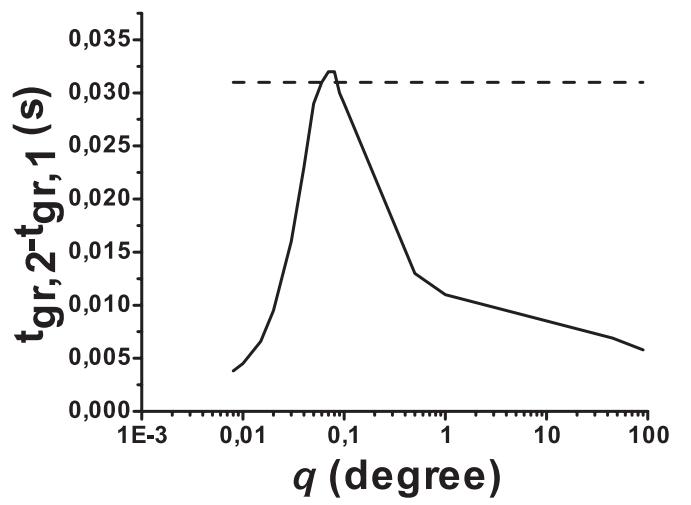

Fig. 6. As in Fig. 5, but for the active region model described be relations (8) and (10).

on spectrograms, even if the agent generating waves is propagating towards lower densities. It happens when $t_{\mathrm{gr}, 2}-t_{\mathrm{gr}, 1}>\left|\left(s_{0}\left(\omega_{2}\right)-s_{0}\left(\omega_{1}\right)\right) / v_{\mathrm{s}}\right|$. Similar computations were made also for the active region model described by relations (8) and (10). The results are shown in Fig. 6.

\section{Conclusions}

In a solar atmosphere, for concrete events the real density and magnetic field profiles can differ from those assumed. But it was found that the results are only weakly dependent on them, being much more sensitive to types of waves considered, i.e. to the generation mechanism. Thus the obtained results can be used in interpretations of real phenomena.

It was shown that the time delays and their spectra can have various values and forms depending on plasma parameters of the radio wave emission and propagation. It was found that for short time delays it is not necessary to assume small scales of plasma parameter changes in the solar atmosphere. For the emission on the double upper-hybrid frequency a considerable decrease of the time delay is due to an increase of the electron plasma density of the radio wave generation. This result weakly depends on selected models of the magnetic field. For the electron density distribution given by the barometric law, for high values of the density, the time delay can be even decreased to values which are characteristic for spikes $(\approx 100 \mu \mathrm{s})$. In the dense flare plasma, for which $k>2-3$, the power index $a$ is negative. In a diluted plasma $a$ becomes positive, but in this case it is considerably lower than predicted using a simple theoretical model, i.e. $\tau=\left(1 / v_{\mathrm{x}}-1 / v_{\mathrm{o}}\right) \times$ const (with $a=3$ ) and lower than observed values for dm-pulsations $(a=3)$. The best agreement of values presented in the paper by Fleishman et al. (2002) was found for the model with the emission on double gyro-frequency. Other considered mechanisms of the radio emission (on double plasma frequency or double upper-hybrid frequency) do not agree with these results. But, it is necessary to note that due to gyroresonant absorption, in the model with the emission on double gyro-frequency, escape of the observed radiation is possible only for angles $\theta<3-10^{\circ}$. Furthermore, it is shown that the effect of a difference in the group and light velocities on the frequency drift can be important for the wave propagation along magnetic field lines for frequencies close to the plasma frequency. In this case, radiospectrographic structures with positive frequency drift can appear even if the agent generating the waves is propagating towards lower densities.

Acknowledgements. This research was supported by the RFBR grant 02-02-17733 and also by the grant IAA3003202 of the Academy of Sciences of the Czech Republic.

\section{References}

Allaart, M. A. P., van Nieuwkoop, J., Slottje, C., \& Sender, L. H. 1990, Sol. Phys., 130, 183

Aschwanden, M. J. 1987, Sol. Phys., 111, 113

Benz, A. O., \& Pianezzi, P. 1997, A\&A, 323, 250

Bernstein, I. B. 1958, Phys. Rev., 109, 10

Bogod, V. M., Mercier, C., \& Yasnov, L. V. 2001, J. Geoph. Res., 106, N A11, 25.353

Chernov, G. P., \& Zlobec, P. 1995, Sol. Phys., 160, 79

Dulk, G. A., \& McLean, D. J. 1978, Sol. Phys., 57, 279

Fleishman, G. D., Fu, Q. J., Huang, G.-L., Melnikov, V. F., \& Wang, M. 2002, A\&A, 385, 671

Fu, Q., Ji, H., Liu, Y., et al. 2000, Astrophys. Rep., 36, 1

Ginzburg, V. L., \& Zheleznyakov, V. V. 1958, Astron. Zhurnal, 35, 694

Gudel, M., \& Benz, A. O. 1988, A\&AS, 75, 243

Gurman, J. B., \& House, L. L. 1981, Sol. Phys., 71, 5

Isliker, H., \& Benz, A. O. 1994, A\&A, 104, 145

Jiřička, K., Karlický, M., Mészárosová, H., \& Snížek, V. 2001, A\&A, 375,243

Kuijpers, J. 1975, Sol. Phys., 44, 173

Melnikov, V. F., Fleishman, G. D., Fu, Q. J., \& Huang, G.-L. 2002, Astronomical Zh., 79, 551 (in Russian)

Melrose, D. B. 1970, Austr. J. Phys., 23, 871

Mollwo, L. 1983, Sol. Phys., 83, 305

Newkirk, G. Jr. 1967, ARA\&A, 5, 213

Slottje, C. 1981, Atlas of fine structures of dynamic spectra of solar type IV-dm and some type II bursts, Utrecht Observatory

Wang, M., Fu, Q., Xie, R., \& Huang, G.-L. 2000, Astrophys. Rep., 36, 77

Winglee, R. M., \& Dulk, G. A. 1986, ApJ, 307, 808

Zheleznyakov, V. V. 1977, Electromagnetic Waves in Cosmic Plasma, 482

Zheleznayakov, V. V. 1996, Radiation in Astrophysical Plasmas (Dordrecht, The Netherlands: Kluwer)

Zheleznyakov, V. V., \& Zlotnik, E. Ya. 1975, Sol. Phys., 44, 447

Zlobec, P., \& Karlický, M. 1998, Sol. Phys., 182, 477 